\title{
Sleep Disorders over the Full Range of Chronic Kidney Disease
}

\author{
Andreas Pierratos $^{\mathrm{a}}$ Patrick J. Hanly ${ }^{\mathrm{b}}$ \\ a Department of Nephrology, Humber River Regional Hospital, University of Toronto, Toronto, Ont., and \\ bSleep Centre, Foothills Medical Centre, University of Calgary, Calgary, Alta., Canada
}

\section{Key Words}

Sleep $\cdot$ End-stage renal disease $\cdot$ Sleep apnea $\cdot$ Dialysis

\begin{abstract}
Sleep disorders are common and underrecognized in patients at all stages of chronic kidney disease. They include sleep apnea, insomnia, excessive sleepiness, restless legs syndrome and periodic limb movement disorder. They can be related to underlying uremia or comorbidities. Sleep disorders can affect the quality of life, and some are associated with increased morbidity and mortality. Clinical assessment, polysomnography and other standardized assessments are required for diagnosis. Therapeutic approaches include improvement in uremia management, treatment of comorbidities or specific interventions directed at individual sleep disorders. Diagnosis and treatment of sleep disorders in this population may improve quality of life and patient survival.
\end{abstract}

Copyright $\odot 2011$ S. Karger AG, Basel

\section{Introduction}

Sleep disorders are common and under-recognized in patients at all stages of chronic kidney disease (CKD). A review of 17 studies in patients with end-stage renal disease (ESRD) indicated that 'sleep disturbance' was com- mon with a mean prevalence of $44 \%$. This review focuses on sleep apnea, insomnia, excessive sleepiness, restless legs syndrome (RLS) and periodic limb movement (PLM) disorder.

\section{Sleep Apnea}

Sleep apnea, in which patients stop breathing during sleep is classified as obstructive (OSA) due to intermittent closure of the upper airway or central due to intermittent loss of respiratory drive. Its severity is defined by the apnea-hypopnea index during a sleep study (polysomnography).

More than $50 \%$ of ESRD patients have sleep apnea $[1,2]$, which is dramatically higher than the general population $(2-4 \%)$ and is similar in predialysis, peritoneal dialysis or hemodialysis patients $[1,3]$. The predominant type of sleep apnea found in patients with ESRD is OSA.

Sleep apnea is caused both by destabilization of central ventilatory control and upper airway occlusion during sleep. Enhanced ventilatory sensitivity to hypercapnia positively correlated with apnea severity [4], and a reduction in ventilatory sensitivity to hypercapnia following conversion from conventional hemodialysis (CHD) to nocturnal hemodialysis (NHD) was associated with a reduction in the severity of OSA [5]. Upper airway occlu-

\section{KARGER \\ Fax +4161306 1234 \\ E-Mail karger@karger.ch}

www.karger.com
(C) 2011 S. Karger AG, Basel

0253-5068/11/0313-0146\$38.00/0

Accessible online at:

www.karger.com/bpu
Andreas Pierratos

112 Joicey Blvd

Toronto, ON M5M 2T6 (Canada)

Tel./Fax +1 4166572669

E-Mail a.pierratos@utoronto.ca 
sion can be caused by fluid overload and interstitial edema in the upper airway [6]. Displacement of fluid from the lower limbs increases neck circumference and pharyngeal resistance [7] and reduces upper airway crosssectional area, contributing to the pathogenesis of OSA. Pharyngeal cross-sectional area measured with acoustic pharyngometry in patients on CHD was smaller than in controls, suggesting that this predisposes them to upper airway occlusion during sleep [8]. Conversion of ESRD patients from CHD to NHD was associated with an increase in pharyngeal cross-sectional area [9], possibly due to improved fluid removal. Conversion from continuous ambulatory peritoneal dialysis (CAPD) to nocturnal peritoneal dialysis has also been shown to reduce the frequency of OSA [10] associated with reduced pharyngeal narrowing as measured by magnetic resonance imaging [11]. Upper airway dilator muscle dysfunction due to neuropathy or myopathy associated with chronic uremia or the underlying cause of ESRD such as diabetes mellitus can also cause pharyngeal narrowing.

Advancing age, male sex and excessive weight are risk factors for the development of OSA in the general population. Some investigators have reported that these associations are weak in the ESRD population $[1,4,12]$. Obesity is not required for ESRD patients to develop OSA, and snoring is less intense than in patients with normal renal function.

Sleep apnea may exacerbate the symptoms of CKD such as daytime fatigue, sleepiness and impaired neurocognitive function. Sleep apnea may exacerbate the cardiovascular complications of ESRD. In patients with ESRD, hypoxemia during sleep is associated with nocturnal hypertension, left ventricular hypertrophy, impaired sympathovagal balance and an increased risk of cardiovascular complications. Coexisting sleep apnea may increase the risk of death in this patient population [13].

Although the characteristic clinical features of sleep apnea may be absent, a history of snoring, witnessed apneas during sleep and daytime sleepiness are suggestive of sleep apnea. Subjective sleepiness can be assessed with a number of simple scales, such as the Epworth Sleepiness Scale. Tools to estimate the likelihood of sleep apnea include the Berlin questionnaire and adjusted neck circumference, but these have not been validated in the ESRD population. Objective diagnostic testing can be limited to ambulatory monitoring at home (which typically records airflow, snoring, respiratory movement, oxygen saturation and heart rate). Alternatively, polysomnography can be performed in a sleep laboratory, which includes comprehensive monitoring of respiration, sleep and leg move- ments. Additional diagnostic tests such as the Multiple Sleep Latency Test (MSLT) and the Maintenance of Wakefulness Test can be considered for the evaluation of daytime sleepiness.

Sleep apnea should be treated if the patient has symptoms such as fragmented sleep and daytime sleepiness that are due to this disorder. In patients without sleeprelated symptoms, consideration should be given to treating sleep apnea in those with severe disease (apnea-hypopnea index $>30$ ) since OSA of this severity has been associated with increased cardiovascular morbidity and mortality. Finally, sleep apnea should be treated if it is thought to be exacerbating co-existing medical disorders such as hypertension, myocardial ischemia and respiratory failure.

Treatment options include lifestyle modification (weight loss, avoidance of alcohol consumption close to bedtime, and avoidance of the supine position during sleep), the use of a dental appliance or continuous positive airway pressure to keep the upper airway open during sleep, and surgical correction of upper airway obstruction, such as removal of very enlarged tonsils, which is infrequent in adults. The experience from clinical practice suggests that ESRD patients have poor compliance with continuous positive airway pressure therapy, which is most likely due to the multifactorial etiology of sleep disruption in this patient population. Sleep apnea may be improved by changing the mode of renal replacement therapy. Although sleep apnea is not corrected by CHD or peritoneal dialysis, NHD, which enables patients to receive hemodialysis at home during sleep, has been shown to improve sleep apnea. Similar results have been reported in the CAPD population with nocturnal cycler-assisted peritoneal dialysis [10]. This was attributed to more effective fluid removal and its impact on the upper airway during sleep [11]. Although case reports have indicated correction of sleep apnea following successful kidney transplantation [14], results from case series are mixed, with some indicating that sleep apnea improves [15] and others indicating that sleep apnea resolves in only a minority of patients following transplantation [12].

\section{Insomnia}

Diagnosis is based on clinical assessment, with chronic complaints of difficulty initiating or maintaining sleep, waking up too early or unrefreshing sleep, despite adequate opportunity and circumstances for sleep, leading to impairment of daytime function. 
Insomnia is common in patients with ESRD who are treated with CHD or CAPD (38-71\%) [16-18] without a significant difference between the two.

The etiology of insomnia is multifactorial, and includes sleep disruption associated with RLS, PLM disorder and sleep apnea $[19,20]$, metabolic factors, bone pain and pruritus, psychiatric disorders such as depression, circadian rhythm disorders such as delayed sleep phase syndrome, medications, and poor sleep hygiene including frequent napping during daytime dialysis.

Polysomnographic studies have reported a total sleep time of 4.4-6 h per night, fragmented by a high frequency of arousals (up to $30 / \mathrm{h}$ ), resulting in a sleep efficiency that ranged from 66 to $85 \%[19,21,22]$. Stage 1 and 2 nonrapid eye movement sleep is often increased, whereas slow-wave sleep and REM sleep are reduced $[19,23]$.

Insomnia is a significant source of stress, which impairs quality of life [17] but has also been associated with shorter survival in patients on CHD and CAPD [24].

Clinical assessment and questionnaires can be used to quantify the severity of sleep-related symptoms. Objective sleep testing is generally reserved when comorbid sleep disorders such as sleep apnea, RLS and PLM, are suspected. The clinical assessment includes history of medical and psychiatric comorbidities, medications, alcohol and caffeine consumption. Completion of a 2-week sleep diary may help. Questionnaires such as the Pittsburgh Sleep Quality Index (PSQI), and the Insomnia Severity Index can be used. Objective testing of sleep can be performed by techniques including actigraphy, ambulatory monitoring and polysomnography.

Therapeutic options include: (1) correction of underlying medical conditions including ESRD, (2) nonpharmacologic strategies to consolidate nocturnal sleep, and (3) medications to improve sleep-related symptoms. There have been a few studies that have evaluated the impact of renal function replacement on insomnia. Novak et al. [25] found that sleep-related symptoms component of the Pittsburgh Symptom Score, improved significantly in 45 patients who were started on CAPD. An RCT comparing automated peritoneal dialysis with CAPD included a subjective description of 'sleep quality' [26], but no differences in measures of sleep quality were found. In a study which reported that NHD improved sleep apnea, there was no improvement in polysomnographic features of sleep fragmentation [19]. The prevalence of poor sleep, reflected by the PSQI, has been reported as $30 \%$ in a cohort of 115 patients with renal transplantation, but sleep quality before transplantation was not reported [27].
In a study of 24 CAPD patients, cognitive behavioral therapy was associated with a small but significant improvement in the PSQI score compared to the control group [28]. Although a variety of hypnotic and sedating medications are commonly used in the management of chronic insomnia, there have been no appropriately designed studies to evaluate their safety and efficacy in patients with ESRD.

\section{Excessive Sleepiness}

Excessive sleepiness is defined as the inability to stay awake or alert during the major waking episodes of the day, resulting in unintended lapses into drowsiness or sleep.

Sixty-six percent of patients in a CHD unit complained of daytime sleepiness [29]. Sleepiness appears to be equally prevalent in ESRD patients treated with CAPD as in those receiving $\mathrm{CHD}$.

Hanly et al. [21] evaluated 24 unselected patients treated with CHD. Subjective sleepiness using the Epworth Sleepiness Scale $(\mathrm{ESS}<8)$ was reported in $50 \%$ of patients, and objective sleepiness using the MSLT (sleep latency $<5 \mathrm{~min}$ ) was found in $50 \%$ of patients. Sleepiness was associated with both a higher BUN and a higher frequency of PLM. Parker et al. [22] evaluated 46 selected CHD patients after excluding those with known causes of daytime sleepiness. Despite this screening, 50\% of patients had sleep apnea and 50\% had PLM. Thirteen percent of patients had evidence of 'pathological' sleepiness (sleep latency $<5 \mathrm{~min}$ ). Objective sleepiness correlated with indices of sleep apnea severity and the frequency of arousals on polysomnography [22]. The respiratory disturbance index, a measure of apnea frequency, accounted for only $11 \%$ of the variance in the measures of sleepiness, which implies that nonrespiratory factors play a significant role. In addition, $48 \%$ of patients had REM sleep on at least one nap, and $17 \%$ of patients had REM sleep on two or more naps, which may reflect the severity of night-time sleep disruption or may indicate a basic desynchronization of the timing of REM sleep.

Daytime sleepiness can be quantified clinically as falling asleep involuntarily in either passive (e.g. reading, watching TV) or active (e.g. driving, during conversation) situations. Sleepiness can be further assessed by questionnaires such as the Epworth Sleepiness Scale and the Stanford Sleepiness Score and by laboratory tests such as the MSLT and the Maintenance of Wakefulness Test. 
The consequences of daytime sleepiness can range from decreased productivity at work to an increased risk of motor vehicle accidents.

Treatment of excessive sleepiness is primarily directed at the underlying cause, which can include clinical entities such as insufficient sleep, sleep disruption from a coexisting sleep disorder such as sleep apnea, RLS, PLM and narcolepsy, side effects of sedating medications, and chronic medical and psychiatric disorders. If the cause is unclear (idiopathic hypersomnolence) and the patient has disabling sleepiness, stimulant medications such as modafinil, methylphenidate and amphetamines may be considered. The impact of NHD on sleepiness in patients with ESRD has been evaluated [21]. Fifteen ESRD patients had overnight polysomnography and MSLT before and after they were converted from CHD to NHD. The data suggested that sleepiness improves following conversion to NHD, possibly related to reduced sleep disruption associated with PLM.

\section{RLS and PLM Disorder}

RLS refers to complaints of an unpleasant sensation in the legs accompanied by an urge to move them, typically in the evening or early part of the night, that is worse during periods of inactivity and transiently relieved by movement [30]. PLM disorder refers to involuntary jerking movement of the legs (and occasionally the arms) during sleep that may be disruptive to the patient or bed-partner. RLS is almost always associated with PLM disorder, but PLM can occur in the absence of RLS.

RLS has been reported in $14-23 \%$ in patients on CHD [31] and 20-57\% in CKD patients [30]. The prevalence of PLM is greater than $50 \%$ in the CHD and CAPD population $[19,21,22]$ associated with sleep onset and sleep maintenance insomnia [18].

The pathogenesis of RLS and PLM in patients with ESRD is not understood. Common risk factors include anemia, iron deficiency, elevated serum calcium and peripheral and central nervous system abnormalities. In addition, it is likely that alteration of dopamine and opioid activity in the nervous system plays a role. Correction of anemia by treatment with erythropoietin or intravenous iron was associated with a significant improvement in RLS and PLM. Peripheral neuropathy, secondary to uremia or the underlying cause of ESRD (such as diabetes) may also predispose patients to develop RLS and/or PLM.
RLS associated with ESRD appears to progress more rapidly, become more severe and is less responsive to dopaminergic medications than idiopathic RLS.

RLS is associated with difficulty initiating sleep, poor sleep quality and impaired health-related quality of life $[31,32]$. Furthermore, RLS has been associated with depression in patients with CKD [33]. These sleep disorders have also been associated with increased mortality in patients with ESRD [32, 34].

RLS is diagnosed clinically. PLM disorder is diagnosed by polysomnography, which reveals periodic, involuntary movements of the legs during sleep. In order to determine whether PLM are responsible for complaints of sleep disruption, a trial of pharmacologic therapy may be required.

The indications to treat RLS and PLM are based on the severity of associated symptoms. There is no indication to treat asymptomatic PLM. General treatment measures include reduction of potential exacerbating factors such as excess caffeine, alcohol and nicotine, medical conditions (anemia, iron deficiency), and medications (tricyclic antidepressants, serotonin reuptake inhibitors, dopamine antagonists). Medications used to treat RLS and PLM include L-dopa and dopamine agonists such as pramipexole. These are favored over benzodiazepines such as clonazepam and opiates. Gabapentin may also be considered as an alternative medication. The frequency of PLM did not change when patients were converted from CHD to NHD [19]. However, kidney transplantation has been associated with an improvement in both RLS and PLM in several small studies [35, 36].

\section{Conclusion}

Sleep disorders are very common in patients with ESRD. Recognition and treatment of these disorders has the potential to both improve the quality of life and decrease morbidity and mortality in this patient population.

\section{Disclosure Statement}

The authors declare no conflict of interest. 


\section{References}

$\checkmark 1$ Kimmel PL, Miller G, Mendelson WB: Sleep apnea syndrome in chronic renal disease. Am J Med 1989;86:308-314.

-2 Unruh ML, Sanders MH, Redline S, Piraino BM, Umans JG, Hammond TC, Sharief I, Punjabi NM, Newman AB: Sleep apnea in patients on conventional thrice-weekly hemodialysis: comparison with matched controls from the Sleep Heart Health Study. J Am Soc Nephrol 2006;17:3503-3509.

$\checkmark 3$ Wadhwa NK, Mendelson WB: A comparison of sleep-disordered respiration in ESRD patients receiving hemodialysis and peritoneal dialysis. Adv Perit Dial 1992;8:195-198.

$\checkmark 4$ Beecroft J, Duffin J, Pierratos A, Chan CT, McFarlane P, Hanly PJ: Enhanced chemoresponsiveness in patients with sleep apnoea and end-stage renal disease. Eur Respir J 2006;28:151-158.

5 Beecroft JM, Duffin J, Pierratos A, Chan CT, McFarlane P, Hanly PJ: Decreased chemosensitivity and improvement of sleep apnea by nocturnal hemodialysis. Sleep Med 2009; 10:47-54.

6 Anastassov GE, Trieger N: Edema in the upper airway in patients with obstructive sleep apnea syndrome. Oral Surg Oral Med Oral Pathol Oral Radiol Endod 1998;86:644-647.

$\checkmark 7$ Chiu KL, Ryan CM, Shiota S, Ruttanaumpawan P, Arzt M, Haight JS, Chan CT, Floras JS, Bradley TD: Fluid shift by lower body positive pressure increases pharyngeal resistance in healthy subjects. Am J Respir Crit Care Med 2006;174:1378-1383.

$>8$ Beecroft JM, Hoffstein V, Pierratos A, Chan CT, McFarlane PA, Hanly PJ: Pharyngeal narrowing in end-stage renal disease: implications for obstructive sleep apnoea. Eur Respir J 2007;30:965-971.

-9 Beecroft JM, Hoffstein V, Pierratos A, Chan CT, McFarlane P, Hanly PJ: Nocturnal haemodialysis increases pharyngeal size in patients with sleep apnoea and end-stage renal disease. Nephrol Dial Transplant 2008;23: 673-679.

-10 Tang SC, Lam B, Ku PP, Leung WS, Chu CM, Ho YW, Ip MS, Lai KN: Alleviation of sleep apnea in patients with chronic renal failure by nocturnal cycler-assisted peritoneal dialysis compared with conventional continuous ambulatory peritoneal dialysis. J Am Soc Nephrol 2006;17:2607-2616.

-11 Tang SC, Lam B, Lai AS, Pang CB, Tso WK, Khong PL, Ip MS, Lai KN: Improvement in sleep apnea during nocturnal peritoneal dialysis is associated with reduced airway congestion and better uremic clearance. Clin J Am Soc Nephrol 2009;4:410-418.

-12 Beecroft JM, Zaltzman J, Prasad R, Meliton G, Hanly PJ: Impact of kidney transplantation on sleep apnoea in patients with endstage renal disease. Nephrol Dial Transplant 2007;22:3028-3033.
13 Tang SC, Lam B, Yao TJ, Leung WS, Chu CM, Ho YW, Ip MS, Lai KN: Sleep apnea is a novel risk predictor of cardiovascular morbidity and death in patients receiving peritoneal dialysis. Kidney Int 2010;77:1031-1038.

14 Auckley DH, Schmidt-Nowara W, Brown LK: Reversal of sleep apnea hypopnea syndrome in end-stage renal disease after kidney transplantation. Am J Kidney Dis 1999; 34:739-744.

15 Lee JJ, Kim GS, Kim JA, Kim SJ, Kang JG, Kim GH, Jung HH: Improvement of sleeprelated breathing disorder in patients with end-stage renal disease after kidney transplantation. Clin Transplant 2009, Epub ahead of print.

16 Sabbatini M, Minale B, Crispo A, Pisani A, Ragosta A, Esposito R, Cesaro A, Cianciaruso $\mathrm{B}$, Andreucci VE: Insomnia in maintenance haemodialysis patients. Nephrol Dial Transplant 2002;17:852-856.

17 Iliescu EA, Coo H, McMurray MH, Meers CL, Quinn MM, Singer MA, Hopman WM: Quality of sleep and health-related quality of life in haemodialysis patients. Nephrol Dial Transplant 2003;18:126-132.

18 Hui DS, Wong TY, Ko FW, Li TS, Choy DK Wong KK, Szeto CC, Lui SF, Li PK: Prevalence of sleep disturbances in Chinese patients with end-stage renal failure on continuous ambulatory peritoneal dialysis. Am J Kidney Dis 2000;36:783-788.

19 Hanly PJ, Pierratos A: Improvement of sleep apnea in patients with chronic renal failure who undergo nocturnal hemodialysis. N Engl J Med 2001;344:102-107.

20 Loewen A, Siemens A, Hanly P: Sleep disruption in patients with sleep apnea and endstage renal disease. J Clin Sleep Med 2009;5: 324-329.

21 Hanly PJ, Gabor JY, Chan C, Pierratos A: Daytime sleepiness in patients with CRF: impact of nocturnal hemodialysis. Am J Kidney Dis 2003;41:403-410.

-22 Parker KP, Bliwise DL, Bailey JL, Rye DB: Daytime sleepiness in stable hemodialysis patients. Am J Kidney Dis 2003;41:394-402.

-23 Unruh ML, Sanders MH, Redline S, Piraino BM, Umans JG, Chami H, Budhiraja R, Punjabi NM, Buysse D, Newman AB: Subjective and objective sleep quality in patients on conventional thrice-weekly hemodialysis: comparison with matched controls from the sleep heart health study. Am J Kidney Dis 2008;52:305-313.

24 Elder SJ, Pisoni RL, Akizawa T, Fissell R, Andreucci VE, Fukuhara S, Kurokawa K, Rayner HC, Furniss AL, Port FK, Saran R: Sleep quality predicts quality of life and mortality risk in haemodialysis patients: results from the Dialysis Outcomes and Practice Patterns Study (DOPPS). Nephrol Dial Transplant 2008;23:998-1004.
25 Novak MJ, Sheth H, Bender FH, Fried L, Piraino B: Improvement in Pittsburgh Symptom Score index after initiation of peritoneal dialysis. Adv Perit Dial 2008;24:46-50.

26 Bro S, Bjorner JB, Tofte-Jensen P, Klem S, Almtoft B, Danielsen H, Meincke M, Friedberg M, Feldt-Rasmussen B: A prospective, randomized multicenter study comparing APD and CAPD treatment. Perit Dial Int 1999; 19:526-533.

27 Eryilmaz MM, Ozdemir C, Yurtman F, Cilli A, Karaman T: Quality of sleep and quality of life in renal transplantation patients. Transplant Proc 2005;37:2072-2076.

$\checkmark 28$ Chen HY, Chiang CK, Wang HH, Hung KY, Lee YJ, Peng YS, Wu KD, Tsai TJ: Cognitivebehavioral therapy for sleep disturbance in patients undergoing peritoneal dialysis: a pilot randomized controlled trial. Am J Kidney Dis 2008;52:314-323.

$>29$ Walker S, Fine A, Kryger MH: Sleep complaints are common in a dialysis unit. Am J Kidney Dis 1995;26:751-756.

30 Winkelman JW, Chertow GM, Lazarus JM: Restless legs syndrome in end-stage renal disease. Am J Kidney Dis 1996;28:372-378.

31 Mucsi I, Molnar MZ, Ambrus C, Szeifert L, Kovacs AZ, Zoller R, Barotfi S, Remport A, Novak M: Restless legs syndrome, insomnia and quality of life in patients on maintenance dialysis. Nephrol Dial Transplant 2005;20:571-577.

32 Unruh ML, Levey AS, D’Ambrosio C, Fink NE, Powe NR, Meyer KB, Choices for Healthy Outcomes in Caring for End-Stage Renal Disease (CHOICE) Study: Restless legs symptoms among incident dialysis patients: association with lower quality of life and shorter survival. Am J Kidney Dis 2004;43: 900-909.

33 Szentkiralyi A, Molnar MZ, Czira ME, Deak G, Lindner AV, Szeifert L, Torzsa P, Vamos EP, Zoller R, Mucsi I, Novak M: Association between restless legs syndrome and depression in patients with chronic kidney disease. J Psychosom Res 2009;67:173-180.

34 Benz RL, Pressman MR, Hovick ET, Peterson DD: Potential novel predictors of mortality in end-stage renal disease patients with sleep disorders. Am J Kidney Dis 2000;35: 1052-1060.

35 Beecroft JM, Zaltzman J, Prasad GV, Meliton G, Hanly PJ: Improvement of periodic limb movements following kidney transplantation. Nephron Clin Pract 2008;109:c133c139.

36 Molnar MZ, Novak M, Ambrus C, Szeifert L, Kovacs A, Pap J, Remport A, Mucsi I: Restless legs syndrome in patients after renal transplantation. Am J Kidney Dis 2005;45:388396. 